\title{
RESEARCH
}

Open Access

\section{Multivisceral resection for adenocarcinoma of the pancreatic body and tail-a retrospective single-center analysis}

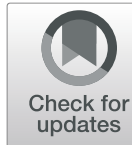

Oliver Beetz, Akin Sarisin, Alexander Kaltenborn, Jürgen Klempnauer, Michael Winkler and Gerrit Grannas* (B)

\begin{abstract}
Background: Adenocarcinoma of the pancreatic body and tail is associated with a dismal prognosis. As patients frequently present themselves with locally advanced tumors, extended surgery including multivisceral resection is often necessary in order to achieve tumor-free resection margins. The aim of this study was to identify prognostic factors for postoperative morbidity and mortality and to evaluate the influence of multivisceral resections on patient outcome.
\end{abstract}

Methods: This is a retrospective analysis of 94 patients undergoing resection of adenocarcinoma located in the pancreatic body and/or tail between April 1995 and December 2016 at our institution. Uni- and multivariable Cox regression analysis was conducted to identify independent prognostic factors for postoperative survival.

Results: Multivisceral resections, including partial resections of the liver, the large and small intestines, the stomach, the left kidney and adrenal gland, and major vessels, were carried out in 47 patients (50.0\%). The median postoperative follow-up time was $12.90(0.16-220.92)$ months.

Median Kaplan-Meier survival after resection was 12.78 months with 1-, 3-, and 5-year survival rates of 53.2\%, 15.8\%, and 9.0\%. Multivariable Cox regression identified coeliac trunk resection ( $p=0.027$ ), portal vein resection ( $p=$ $0.010)$, intraoperative blood transfusions $(p=0.005)$, and lymph node ratio in percentage $(p=0.001)$ as independent risk factors for survival. Although postoperative complications requiring surgical revision were observed more frequently after multivisceral resections (14.9 versus $2.1 \% ; p=0.029$ ), postoperative survival was not significantly inferior when compared to patients undergoing standard distal or subtotal pancreatectomy (12.35 versus 13.87 months; $p=0.377$ ).

Conclusions: Our data indicates that multivisceral resection in cases of locally advanced pancreatic carcinoma of the body and/or tail is justified, as it is not associated with increased mortality and can even facilitate long-term survival, albeit with an increase in postoperative morbidity. Simultaneous resections of major vessels, however, should be considered carefully, as they are associated with inferior survival.

Keywords: Left-sided pancreatic cancer, Distal pancreatectomy, Multivisceral resection, Extended surgery, Lymph node ratio, Pancreatic fistula

\footnotetext{
* Correspondence: Gerrit.Grannas@mh-hannover.de

Department of General, Visceral and Transplant Surgery, Hannover Medical

School, Carl-Neuberg-Strasse 1, 30625 Hannover, Germany
}

C C The Author(s). 2020 Open Access This article is licensed under a Creative Commons Attribution 4.0 International License, which permits use, sharing, adaptation, distribution and reproduction in any medium or format, as long as you give appropriate credit to the original author(s) and the source, provide a link to the Creative Commons licence, and indicate if changes were made. The images or other third party material in this article are included in the article's Creative Commons licence, unless indicated otherwise in a credit line to the material. If material is not included in the article's Creative Commons licence and your intended use is not permitted by statutory regulation or exceeds the permitted use, you will need to obtain permission directly from the copyright holder. To view a copy of this licence, visit http://creativecommons.org/licenses/by/4.0/ The Creative Commons Public Domain Dedication waiver (http://creativecommons.org/publicdomain/zero/1.0/) applies to the data made available in this article, unless otherwise stated in a credit line to the data. 


\section{Background}

Pancreatic adenocarcinoma is a fatal malignant disease with 5 -year survival rates below $10 \%$ and an increasing incidence worldwide [1]. As typical symptoms often occur at a late stage, 80 to $90 \%$ of the patients present themselves with unresectable tumors and dismal prognosis despite efforts of improving non-surgical therapies including chemotherapy regimens $[2,3]$.

In case of resection, severable risk factors impeding long-term survival have been identified in the past, including lymph node metastases, advanced tumor stage, positive resection margins, and distant disease, among others $[4,5]$.

Although the influence of the tumor localization has been a matter of great debate, data from large patient registries have demonstrated that lesions of the pancreatic body and tail, accounting for around 20 to 25\% of the pancreatic adenocarcinomas, are associated with inferior survival, most likely as a result of a delayed diagnosis and a more aggressive tumor biology [6-9]. Distal pancreatectomy is the standard procedure for these tumors; however, advanced tumor stages often require additional resection of extrapancreatic tissue, including large vessels. Data on the effect of such resections is scarce. Recently, Malinka et al. and Panzeri et al. each published results of single-center studies, indicating that multivisceral resections are justifiable in selected patients $[10,11]$. However, especially the role of vascular resections remains to be elusive. Recent recommendations, for example, discourage arterial resections of the coeliac axis without prior neoadjuvant chemotherapy, whereas venous resections are generally regarded as feasible and safe. Nonetheless, reports are contradictory throughout the available literature, not least because data of patients with pancreatic head and body/tail lesions are often pooled $[12,13]$.

The aim of our study was to evaluate the influence of different types of multivisceral resections, among other potential prognostic factors, on postoperative morbidity and mortality in patients with adenocarcinoma of the pancreatic body and/or tail.

\section{Methods}

\section{Study design and patient cohort}

This is a retrospective, single-center analysis of 94 patients with adenocarcinoma of the pancreatic body and/ or tail undergoing surgery between April 1995 and December 2016 at the Department of General, Visceral and Transplant Surgery, Hannover Medical School, in Hannover, Germany.

\section{Inclusion and exclusion criteria}

Included were all patients with intraoperatively and histologically confirmed ductal adenocarcinoma of the pancreatic body and/or tail undergoing resection. No exclusion criteria were defined.

\section{Definition of variables}

Multivisceral resections were defined as distal or subtotal pancreatectomy (including lymphadenectomy of the stations 10, 11, and 18 and splenectomy) with additional resection of contiguous or distant organs, including large vessels, also referred to as "extended distal pancreatectomy" and "distal pancreatectomy with resection of non-contiguous organs" according to the International Study Group for Pancreatic Surgery [14, 15].

For classification of medical co-morbidities and preoperative fitness prior surgery, the American Society of Anesthesiologists (ASA) Physical Status Classification System was applied [16].

Preoperative anemia was defined according to the World Health Organization as hemoglobin concentrations lower than $12.0 \mathrm{~g} / \mathrm{dl}$ in female patients and lower than $13.0 \mathrm{~g} / \mathrm{dl}$ in male patients [17].

Surgical complications were defined as postoperative complications requiring surgical revision or intervention.

Postoperative pancreatic fistulas were defined according to the latest definition of the International Study Group for Pancreatic Surgery [18]. Postoperative pancreatic fistulas grade $\mathrm{B}$ and $\mathrm{C}$ were defined as clinically relevant.

For the classification of the respective tumor stage, the current AJCC/UICC $8^{\text {th }}$ edition was applied [19].

The lymph node ratio was analyzed as continuous variable in percent for regression analysis. For visualization of postoperative survival, the lymph node ratio was analyzed as binary variable (cut-off $\geq 20 \%$ ).

Follow-up time was defined as time between surgery and last contact or death.

Survival times are reported as Kaplan-Meier median estimates, unless stated otherwise.

\section{Study endpoints}

The primary endpoint was postoperative survival after pancreatic resection. Secondary endpoints were clinically relevant postoperative pancreatic fistulas and postoperative complications in general.

\section{Statistical analysis}

The influence of nominal and ordinal variables on binary endpoints was analyzed with chi-squared test and Fisher's exact test where appropriate.

Continuous endpoints, such as mean and median values, were compared with the Student's $t$ test in case of normal distribution or the Mann-Whitney $U$ test.

Risk factors for postoperative survival were analyzed with Cox regression analysis. Independent risk factors were identified by purposeful selection of variables with 
a rate of missing values of $<10 \%$ and $p$ values in univariable Cox regression of $<0.300$ and consecutive stepwise forward selection. Kaplan-Meier analyses including Logrank tests were performed where appropriate.

The identification of risk factors for clinically relevant postoperative pancreatic fistulas was achieved by univariable and multivariable binary logistic regression analysis, as described above.

Statistical significance was set at a $p$ value of $<0.050$ and is shown in bold (tables) or marked with an asterisk (figures).

The collected data was implemented and analyzed using SPSS statistical software (version 26; SPSS Inc.; IBM Corporation, Armonk, NY USA), and respective figures were created with GraphPad Prism (version 8.4.0 for Windows, GraphPad Software, La Jolla, CA USA).

\section{Results}

\section{Study cohort and preoperative course}

Females (51.1\%) and males (48.9\%) were equally distributed among the cohort of 94 patients. The median age was 65 (41-84) years.

Patients most commonly presented themselves with epigastric pain (68.1\%), weight loss (39.4\%), and back pain $(20.2 \%)$. Diabetes prior surgery was present in 20 (21.3\%) patients.

Elevated tumor markers (CA19-9 and CEA) were observed in $42(44.7 \%)$ and 19 (20.2\%) patients, respectively, with a high rate of missing values, especially in the early observation period.

Twenty-two (23.4\%) patients showed severe systemic disease and/or substantive functional limitations prior surgery (i.e., ASA score $>2$ ).

A summary of the biometrical and preoperative data is provided in Table 1.

\section{Pancreatic surgery}

Distal pancreatectomy was performed in 85 (90.4\%) patients. Subtotal pancreatectomy was performed in 9 (9.6\%) patients and was neither associated with increased postoperative morbidity (i.e., complications with or without the need of surgical revision; $p \geq 0.050$ ) nor inferior survival (14.36 versus 12.42 months; $p=0.869$ ).

Simultaneous splenectomy was performed in all but one patient, who underwent splenectomy after polytrauma prior to the pancreatic resection.

Due to the invasion of extrapancreatic tissue, 47 (50.0\%) patients underwent multivisceral resections, including partial resections of the liver (12 patients), the large and small intestine (12 and 8 patients, respectively), the stomach (14 patients), the left kidney (5 patients), the left adrenal gland (13 patients), the portal vein (15 patients), and the coeliac trunk (5 patients). Of note, in $21(44.7 \%)$ of these patients, preoperative imaging led to the suspected diagnosis of local invasion of neighboring tissue or distant metastases prior surgery, whereas in 24 (51.1\%) patients, extrapancreatic disease was diagnosed intraoperatively (missing information in 2 (4.3\%) patients). Supplementary Table 1 (Additional file 1) gives an overview of the performed resections. Patients undergoing multivisceral resection were of similar mean age (64.02 versus 66.04 years; $p=0.296)$, but displayed a significantly lower rate of ASA scores $>2(16.2$ versus $39.0 \% ; p=0.023$ ) when compared to patients with standard resection. Mean operation time (188.37 versus $142.87 \mathrm{~min} ; p<0.001$ ), rate of intraoperative blood transfusions (53.2 versus $19.1 \%$; $p=0.001$ ), and mean number of intraoperatively transfused packed red blood cell (PRBC) units (2.23 versus 0.45 units; $p<$ 0.001 ) were significantly elevated in case of multivisceral resection. Postoperative complications requiring surgical revision were observed more frequently after multivisceral resections (14.9 versus $2.1 \%$; $p=0.029$ ), whereas the incidence of other postoperative complications was comparable to all other patients (31.9 versus $38.3 \%$; $p=0.517$ ). Clinically relevant pancreatic fistulas were slightly more frequent after multivisceral resections (30.4 versus $17.4 \% ; p=0.143$ ) and significantly more frequent after simultaneous partial colectomy (50.0 versus $20.0 \% ; p=0.034$ ). Accordingly, multivisceral resections led to a significantly prolonged median postoperative hospital stay (18 versus 13 days; $p=$ 0.007). Despite an increase in postoperative morbidity, the postoperative survival after multivisceral resection was not inferior (12.35 versus 13.87 months; $p=0.377$; Fig. 1) with two patients still alive after 5 years. However, (partial) resections of the coeliac trunk (3.52 versus 13.01 months; $p=0.012$; Fig. 2) or the portal vein (7.56 versus 14.72 months; $p=0.064$; Fig. 3 ) were associated with a trend towards inferior survival. (Table 2).

Intraoperative blood transfusions were necessary in 34 (36.2\%) patients, ranging from 1 to 24 units of PRBC, and were significantly associated with inferior survival (8.81 versus 15.21 months; $p<0.001$; Fig. 4). Of note, preoperative anemia was detected in $14(14.9 \%)$ patients. Neither the rate of intraoperative blood transfusions (57.1 versus $33.3 \% ; p=0.083$ ) nor the mean number of intraoperatively transfused PRBC units (1.50 versus 1.35 units; $p=0.072$ ) were significantly elevated in these patients.

Of note, none of the surgical procedures was carried out minimally invasive.

Table 1 provides further information on surgical details.

\section{Histopathological results}

Locally advanced tumor stage (T stage $\geq 3$ ) was observed in $50(53.2 \%)$ patients and resulted in inferior survival (9.76 versus 17.35 months; $p=0.039$ ). 
Table 1 Descriptive statistics of the study cohort undergoing distal pancreatectomy for adenocarcinoma

\begin{tabular}{|c|c|c|c|c|c|}
\hline Variables & & & $n_{\text {abs }}\left(n_{\%}\right)$ & Mean; Median (Range) & $\begin{array}{l}\text { Missing } \\
\text { values } \mathrm{n}(\%)\end{array}$ \\
\hline \multirow[t]{2}{*}{ Biometrics } & \multicolumn{2}{|l|}{ Female gender } & $48(51.1)$ & & $0(0.0)$ \\
\hline & \multicolumn{2}{|l|}{ Age (in years) } & & $65.0 ; 65.0(41-84)$ & $0(0.0)$ \\
\hline \multirow[t]{21}{*}{ Preoperative Course } & \multirow[t]{8}{*}{ Initial symptoms } & Epigastric pain & $64(68.1)$ & & $4(4.3)$ \\
\hline & & Weight loss & 37 (39.4) & & \\
\hline & & Back pain & 19 (20.2) & & \\
\hline & & Inappetence & $13(13.8)$ & & \\
\hline & & Nausea & $12(12.8)$ & & \\
\hline & & Vomiting & $6(6.4)$ & & \\
\hline & & Fatigue & $6(6.4)$ & & \\
\hline & & Others & $29(30.9)$ & & \\
\hline & \multirow[t]{3}{*}{ ASA score } & 1 & $7(7.4)$ & & $16(17.0)$ \\
\hline & & 2 & $49(52.1)$ & & \\
\hline & & 3 & $22(23.4)$ & & \\
\hline & Diabetes & & $20(21.3)$ & & $3(3.2)$ \\
\hline & \multirow[t]{8}{*}{ Diagnostics } & Hemoglobin (in g/dl) & & $13.4 ; 13.3(9.9-17.2)$ & $2(2.1)$ \\
\hline & & Anemia & $14(14.9)$ & & \\
\hline & & Platelets (in $10^{3}$ per $\mu \mathrm{l}$ ) & & $237.85 ; 236(56-551)$ & $2(2.1)$ \\
\hline & & Elevated CA19-9 & $42(44.7)$ & & $31(33.0)$ \\
\hline & & Elevated CEA & 19 (20.2) & & $34(36.2)$ \\
\hline & & CT scan & $82(87.2)$ & & $4(4.3)$ \\
\hline & & MRI scan & $5(5.3)$ & & $4(4.3)$ \\
\hline & & Ultrasound & $74(78.7)$ & & $13(13.8)$ \\
\hline & $\begin{array}{l}\text { Preoperative hospital stay } \\
\text { (in days) }\end{array}$ & & & $2.82 ; 2(1-20)$ & $1(1.1)$ \\
\hline \multirow[t]{16}{*}{ Surgical details } & \multirow[t]{2}{*}{ Type of pancreatectomy } & Distal & $85(90.4)$ & & $0(0.0)$ \\
\hline & & Subtotal & $9(9.6)$ & & \\
\hline & Splenectomy & & $93(98.9)$ & & $0(0.0)$ \\
\hline & Multivisceral resection & & $47(50.0)$ & & $0(0.0)$ \\
\hline & \multirow{8}{*}{$\begin{array}{l}\text { Including (partial) resection } \\
\text { of }\end{array}$} & Liver & $12(12.8)$ & & \\
\hline & & Large intestine & $12(12.8)$ & & \\
\hline & & Small intestine & $8(8.5)$ & & \\
\hline & & Stomach & $14(14.9)$ & & \\
\hline & & Kidney & $5(5.3)$ & & \\
\hline & & Adrenal gland & $13(13.8)$ & & \\
\hline & & Coeliac trunk & $5(5.3)$ & & \\
\hline & & Portal vein & $15(16.0)$ & & \\
\hline & Intraoperative PRBC & & $34(36.2)$ & & $0(0.0)$ \\
\hline & Intraoperative PRBC (n) & & & $1.34 ; 0(0-24)$ & \\
\hline & Intraoperative FFP & & $15(16.0)$ & & $0(0.0)$ \\
\hline & Operation time (in minutes) & & & $165.62 ; 155(68-360)$ & $2(2.1)$ \\
\hline \multirow[t]{4}{*}{ Histopathological results } & \multirow[t]{4}{*}{ Tumor localization } & Tail & $42(44.7)$ & & $0(0.0)$ \\
\hline & & Body & $40(42.6)$ & & \\
\hline & & Tail and body & $12(12.8)$ & & \\
\hline & & Including body & $52(55.3)$ & & \\
\hline
\end{tabular}


Table 1 Descriptive statistics of the study cohort undergoing distal pancreatectomy for adenocarcinoma (Continued)

\begin{tabular}{|c|c|c|c|c|c|}
\hline \multirow[t]{2}{*}{ Variables } & & & $n_{\text {abs }}\left(n_{\%}\right)$ & Mean; Median (Range) & $\begin{array}{l}\text { Missing } \\
\text { values } \mathrm{n}(\%)\end{array}$ \\
\hline & \multicolumn{2}{|l|}{$\begin{array}{l}\text { Invasion of peripancreatic } \\
\text { tissue }\end{array}$} & $78(83.0)$ & & $0(0.0)$ \\
\hline & \multirow[t]{6}{*}{ T staging } & Tumor size (in cm) & & $4.79 ; 4.5(1.0-14.0)$ & $0(0.0)$ \\
\hline & & 1 & $7(7.4)$ & & $0(0.0)$ \\
\hline & & 2 & $37(39.4)$ & & \\
\hline & & 3 & $45(47.9)$ & & \\
\hline & & 4 & $5(5.3)$ & & \\
\hline & & T stage $\geq 3$ & $50(53.2)$ & & \\
\hline & \multirow[t]{8}{*}{ Lymph node status } & Lymph nodes ( $n$ total) & & $11.86 ; 10(1-36)$ & $3(3.2)$ \\
\hline & & Lymph nodes (n positive) & & $1.22 ; 0(0-8)$ & \\
\hline & & Lymph node ratio (in \%) & & $11.68 ; 0(0-100)$ & \\
\hline & & Lymph node ratio $\geq 20 \%$ & $24(25.5)$ & & \\
\hline & & N 0 stage & $49(52.1)$ & & $2(2.1)$ \\
\hline & & N 1 stage & $34(36.2)$ & & \\
\hline & & N 2 stage & $9(9.6)$ & & \\
\hline & & $\geq$ N 1 stage & $43(45.7)$ & & \\
\hline & \multicolumn{2}{|l|}{ M 1 stage } & $19(20.2)$ & & $0(0.0)$ \\
\hline & \multirow[t]{3}{*}{ Grading } & 1 & $1(1.1)$ & & $0(0.0)$ \\
\hline & & 2 & $56(59.6)$ & & \\
\hline & & 3 & $37(39.4)$ & & \\
\hline & \multirow[t]{4}{*}{ Resection margin } & 0 & $61(64.9)$ & & $0(0.0)$ \\
\hline & & 1 & $26(27.7)$ & & \\
\hline & & 2 & $7(7.4)$ & & \\
\hline & & $\geq R 1$ & $33(35.1)$ & & \\
\hline & \multirow{7}{*}{$\begin{array}{l}\text { AJCC/UICC classification } \\
\left(8^{\text {th }} \text { ed. }\right)\end{array}$} & la & $6(6.4)$ & & $2(2.1)$ \\
\hline & & $\mathrm{lb}$ & $15(16.0)$ & & \\
\hline & & Ila & $16(17.0)$ & & \\
\hline & & $11 \mathrm{~b}$ & $24(25.5)$ & & \\
\hline & & III & $12(12.8)$ & & \\
\hline & & IV & $19(20.2)$ & & \\
\hline & & $\geq \| I$ & $31(33.0)$ & & \\
\hline
\end{tabular}

ASA American Society of Anesthesiologists, CT computed tomography, MRI magnetic resonance imaging, PRBC packed red blood cells, FFP fresh frozen plasma, AJCC American Joint Committee on Cancer, UICC Union for International Cancer Control

Lymph node metastases were identified in 43 $(45.7 \%)$ cases. Positive nodal status (N1/N2) was significantly associated with inferior survival (10.55 versus 15.64 months; $p=0.015)$ as were positive lymph node ratios of $\geq 20 \%$ (10.25 versus 14.72 months; $p=0.027$; Fig. 5).

Distant metastases were diagnosed in 19 (20.2\%) patients and did not significantly impact on postoperative survival (10.78 versus 13.87 months; $p=0.217$ ), whereas advanced AJCC/UICC stages ( $\geq 3$; 31 (33.0\%) patients) were significantly associated with inferior survival (10.55 versus $15.21 ; p=0.010)$.
Positive resection margins (R1/R2) were confirmed in 33 (35.1\%) patients and were also significantly associated with inferior survival (10.22 versus 15.64 months; $p=0.004)$.

Table 1 summarizes the histopathological data of the study cohort.

\section{Postoperative outcome}

Median follow-up after pancreatic resection was 12.90 (0.16-220.92) months.

Patients stayed in hospital for a median of 15 (5-109) days. 


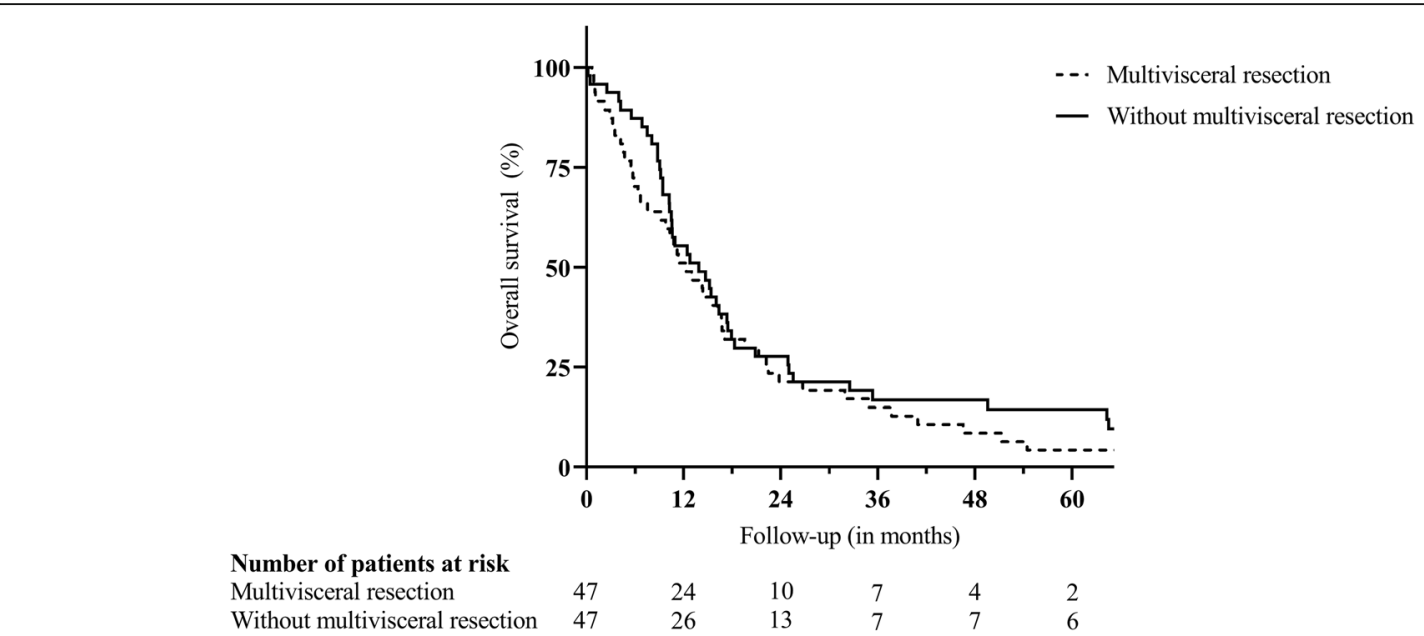

Fig. 1 Postoperative survival after standard distal pancreatectomy or multivisceral resection for ductal adenocarcinoma. Legend: $p=0.377$

We observed postoperative complications in 36 (38.3\%) patients. Surgical revision was required in 8 (8.5\%) cases. Clinically relevant pancreatic fistulas were observed in $22(23.4 \%)$ patients resulting in a prolonged removal of abdominal drains (17 patients) or surgical revision (5 patients).

Six (6.4\%) patients died in the postoperative course. Three patients died due to severe bleeding (two patients) and gastric perforation with consecutive sepsis (one patient) as a result of pancreatic fistula. One patient died after severe bleeding caused by a postoperative intraabdominal abscess, and two further patients died after pulmonary embolism with consecutive multiple organ failure.
The median estimated survival was 12.78 months with 1-, 3-, and 5-year survival rates of 53.2\%, 15.8\%, and 9.0\%. Eighty-eight (93.6\%) patients were deceased at the time of analysis. Table 2 summarizes selected variables regarding the postoperative course and outcome of the study cohort.

\section{Risk factors for clinically relevant pancreatic fistulas}

Univariable regression analysis identified simultaneous partial colectomy as risk factor for the incidence of clinically relevant pancreatic fistulas $(\mathrm{OR}=4.000$; CI $95 \%=$ $1.138-14.063 ; p=0.031$ ); however, none of the analyzed variables was an independent risk factor in multivariable analysis (Supplementary Table 2, Additional file 1).

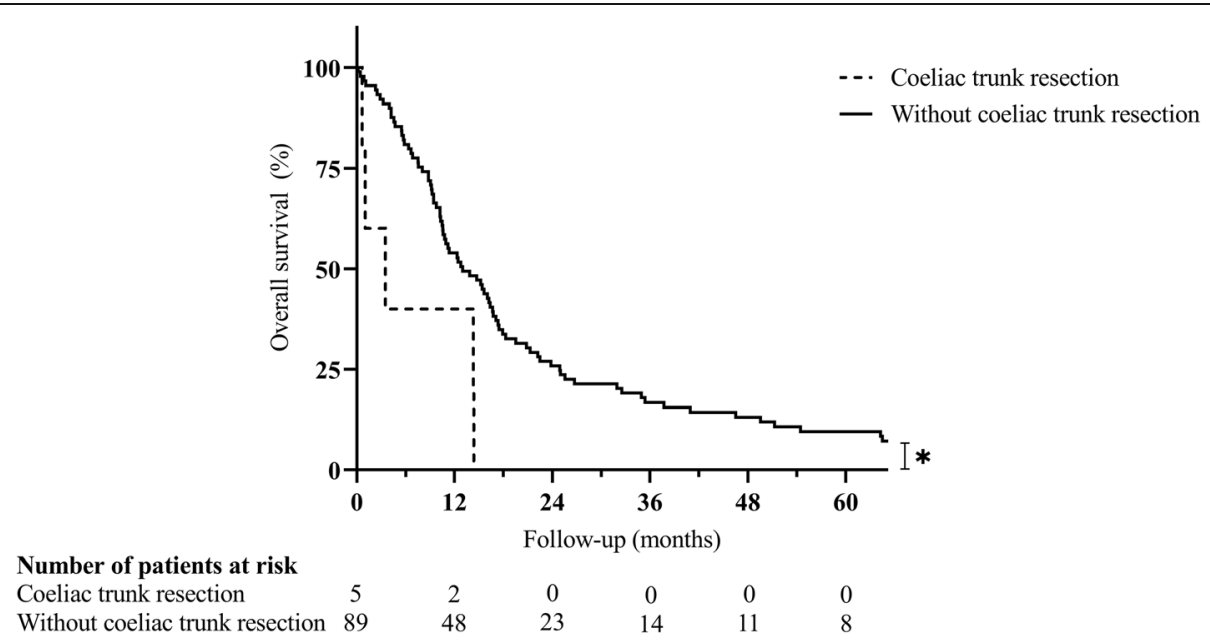

Fig. 2 Survival after distal pancreatectomy for ductal adenocarcinoma in case of coeliac trunk resection. Legend: * $p=0.012$. Statistical significance $(p<0.050)$ is indicated with an asterisk 
Number of patients at risk
Portal vein resection

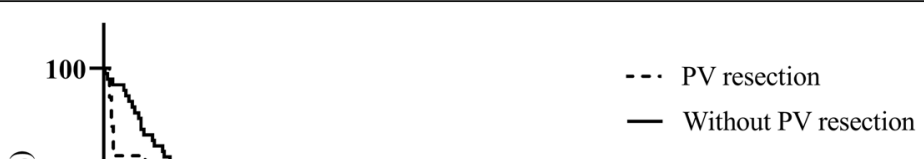

Without portal vein resection

Fig. 3 Survival after distal pancreatectomy for ductal adenocarcinoma in case of portal vein resection. Legend: $p=0.064$. $P V$ portal vein

\section{Risk factors for postoperative survival}

The results of the univariable Cox regression analysis are displayed in Table 3. Multivariable analysis identified coeliac trunk resection $(\mathrm{HR}=3.364$; CI 95\% $=1.147-$ 9.861; $p=0.027)$, portal vein resection $(\mathrm{HR}=2.275$; $\mathrm{CI}$ $95 \%=1.221-4.236 ; p=0.010)$, intraoperative transfusion of PRBC $(\mathrm{HR}=1.998$; CI $95 \%=1.229-3.247 ; p=$
$0.005)$, and lymph node ratio in percentage ( $\mathrm{HR}=1.022$; CI 95\% $=1.009-1.034 ; p=0.001$ ) as independent risk factors for postoperative survival.

\section{Discussion}

Patients suffering from pancreatic adenocarcinoma continue to have an extremely poor prognosis. In cases

Table 2 Postoperative outcome of the study cohort after distal pancreatectomy

\begin{tabular}{|c|c|c|c|c|}
\hline \multicolumn{2}{|l|}{ Variables } & $n_{\text {abs }}\left(n_{\%}\right)$ & Mean; Median (Range) & Missing values $\mathrm{n}(\%)$ \\
\hline \multicolumn{3}{|c|}{ Hospital stay (in days) } & $19.33 ; 15(5-109)$ & $0(0.0)$ \\
\hline \multicolumn{2}{|c|}{ Complications requiring surgery } & $8(8.5)$ & & $0(0.0)$ \\
\hline \multicolumn{2}{|c|}{$\begin{array}{l}\text { Complications not requiring } \\
\text { surgery }\end{array}$} & $33(35.1)$ & & \\
\hline \multirow[t]{6}{*}{ Pancreatic fistula } & Not measured & $47(50.0)$ & & $2(2.1)$ \\
\hline & No biochemical leak & $17(18.1)$ & & \\
\hline & $\begin{array}{l}\text { Biochemical leak } \\
\text { (former Grade A) }\end{array}$ & $6(6.4)$ & & \\
\hline & Grade B & $17(18.1)$ & & \\
\hline & Grade C & $5(5.3)$ & & \\
\hline & $\geq$ Grade B & $22(23.4)$ & & \\
\hline \multicolumn{2}{|c|}{$\begin{array}{l}\text { Postoperative days until } \\
\text { removal of drains }\end{array}$} & & $12.75 ; 9(3-46)$ & $3(3.2)$ \\
\hline \multicolumn{2}{|c|}{ Pancreatin post-surgery } & $34(36.2)$ & & $7(7.4)$ \\
\hline \multicolumn{2}{|c|}{ In-hospital mortality } & $6(6.4)$ & & $0(0.0)$ \\
\hline \multicolumn{2}{|c|}{ Follow-up time in months } & & $23.18 ; 12.90(0.16-220.92)$ & $0(0.0)$ \\
\hline \multicolumn{2}{|c|}{ Survival in months (Kaplan-Meier) } & & $27.96 ; 12.78$ (n.a.) & $0(0.0)$. \\
\hline \multicolumn{2}{|c|}{ 1-year survival (Kaplan-Meier) } & n.a. (53.2) & & \\
\hline \multicolumn{2}{|c|}{ 3-year survival (Kaplan-Meier) } & n.a. (15.8) & & \\
\hline \multicolumn{2}{|c|}{ 5-year survival (Kaplan-Meier) } & n.a. (9.0) & & \\
\hline \multicolumn{2}{|c|}{ Deceased at time of analysis } & $88(93.6)$ & & $0(0.0)$ \\
\hline
\end{tabular}


Number of patients at risk
Intraoperative PRBC

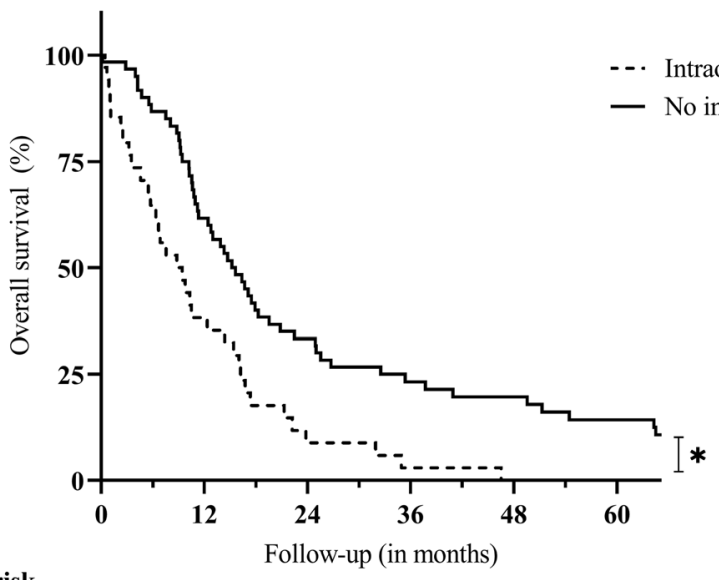

No intraoperative PRBC

$\begin{array}{llcccc}34 & 13 & 3 & 1 & 0 & 0 \\ 60 & 37 & 20 & 13 & 11 & 8\end{array}$

Fig. 4 Survival after distal pancreatectomy for ductal adenocarcinoma in case of intraoperative transfusion of PRBC. Legend: * $p<0.001$. Statistical significance $(p<0.050)$ is indicated with an asterisk. PRBC packed red blood cells

of unresectable disease, median survival of around 7 months has been reported, and although surgical resection is regarded as the only chance for long-term survival, 5-year survival rates after resection of up to around $20 \%$ are still unsatisfying [1,20-22]. Studies investigating prognostic factors for postoperative survival often include patients irrespective of the exact tumor localization, although abundant data suggests that lesions of the pancreatic head differ not only in terms of local tumor extent, invasion of adjacent tissue, and necessary surgical strategy to achieve negative resection margins, but also in tumor biology [21-26].

In order to optimize preoperative patient selection, we have evaluated the effects of multivisceral resections, among other variables, on the postoperative outcome in patients undergoing distal or subtotal pancreatectomy for ductal adenocarcinoma. In summary, multivisceral resections in general were associated with increased morbidity and the risk of reoperation, but not with increased shortor long-term mortality. Although there are currently no

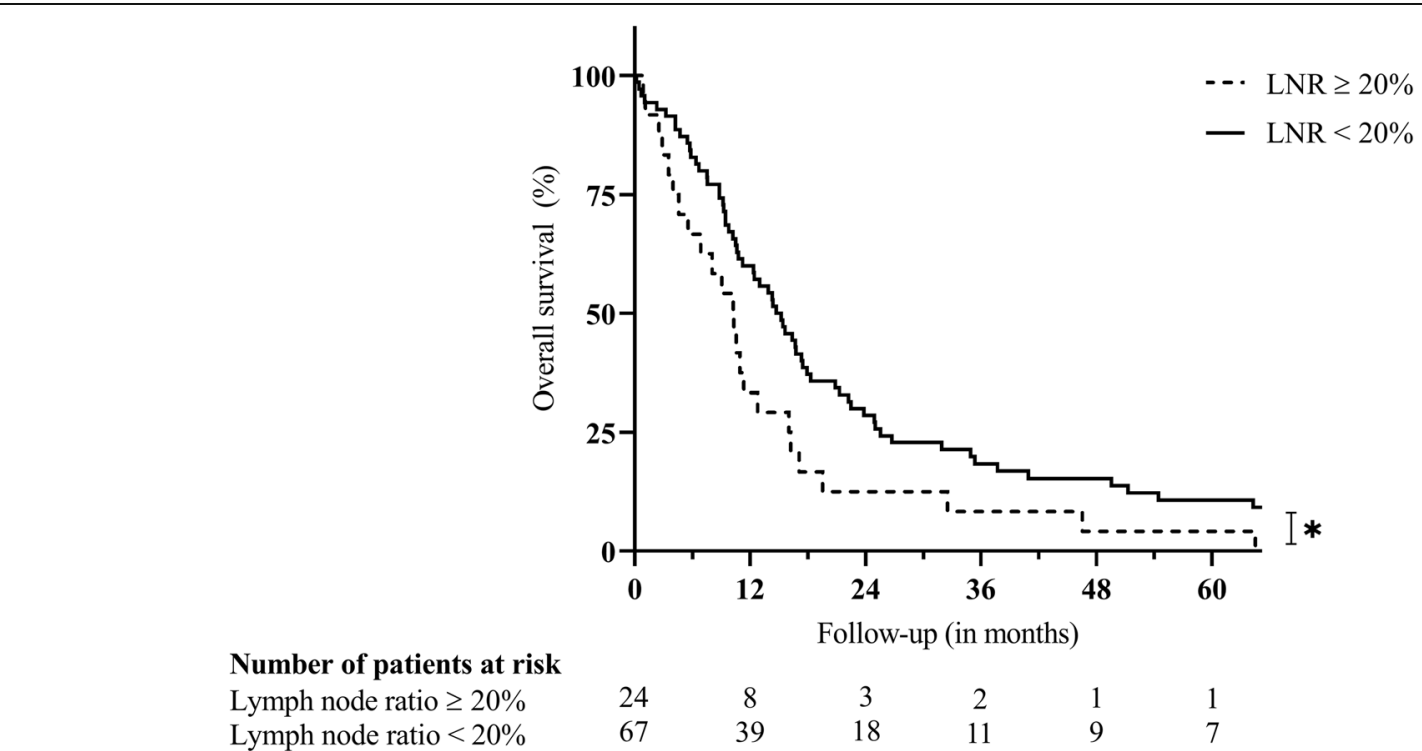

Fig. 5 Survival after distal pancreatectomy for adenocarcinoma in case of lymph node ratio $\geq 20 \%$. Legend: ${ }^{*} p=0.027$. Statistical significance $(p$ $<0.050)$ is indicated with an asterisk. LNR lymph node ratio 
Table 3 Cox regression analysis for identification of risk factors for survival after distal pancreatectomy

\begin{tabular}{|c|c|c|c|c|c|c|c|c|}
\hline \multirow[t]{2}{*}{ Variables } & & & \multicolumn{3}{|c|}{ Univariable analysis } & \multicolumn{3}{|c|}{ Multivariable analysis } \\
\hline & & & $\mathrm{HR}$ & $\mathrm{Cl} 95 \%$ & $p$ value & $\mathrm{HR}$ & $\mathrm{Cl} 95 \%$ & $p$ value \\
\hline \multirow[t]{2}{*}{ Biometrics } & \multicolumn{2}{|l|}{ Female gender } & 0.770 & $0.506-1.171$ & 0.222 & & & \\
\hline & \multicolumn{2}{|l|}{ Age (in years) } & 1.003 & $0.980-1.027$ & 0.805 & & & \\
\hline \multirow{15}{*}{$\begin{array}{l}\text { Preoperative } \\
\text { course }\end{array}$} & \multirow[t]{8}{*}{ Initial symptoms } & Epigastric pain & 0.946 & $0.592-1.512$ & 0.817 & & & \\
\hline & & Weight loss & 1.202 & $0.777-1.861$ & 0.408 & & & \\
\hline & & Back pain & 1.489 & $0.890-2.491$ & 0.130 & & & \\
\hline & & Inappetence & 1.512 & $0.817-2.798$ & 0.188 & & & \\
\hline & & Nausea & 0.702 & $0.362-1.362$ & 0.296 & & & \\
\hline & & Vomiting & 0.881 & $0.356-2.182$ & 0.785 & & & \\
\hline & & Fatigue & 3.007 & $1.279-7.071$ & 0.012 & & & \\
\hline & & Others & 1.060 & $0.666-1.687$ & 0.806 & & & \\
\hline & ASA score $>2$ & & 1.362 & $0.823-2.255$ & 0.229 & & & \\
\hline & Diabetes & & 1.236 & $0.740-2.066$ & 0.419 & & & \\
\hline & \multirow[t]{5}{*}{ Diagnostics } & Hemoglobin (in g/dl) & 0.973 & $0.821-1.154$ & 0.756 & & & \\
\hline & & Anemia & 1.442 & $0.793-2.621$ & 0.231 & & & \\
\hline & & Platelets (in $10^{3}$ per $\mu \mathrm{l}$ ) & 0.996 & $0.993-1.000$ & 0.047 & & & \\
\hline & & Elevated CA19-9 & 1.480 & $0.853-2.568$ & 0.163 & & & \\
\hline & & Elevated CEA & 0.848 & $0.478-1.504$ & 0.572 & & & \\
\hline \multirow[t]{14}{*}{ Surgical details } & Type of pancreatectomy & More than distal & 1.060 & $0.530-2.121$ & 0.869 & & & \\
\hline & Multivisceral resection & & 1.208 & $0.794-1.839$ & 0.378 & & & \\
\hline & \multirow{8}{*}{$\begin{array}{l}\text { Including (partial) } \\
\text { resection of }\end{array}$} & Liver & 1.386 & $0.750-2.562$ & 0.298 & & & \\
\hline & & Large intestine & 1.207 & $0.654-2.228$ & 0.548 & & & \\
\hline & & Small intestine & 1.198 & $0.577-2.485$ & 0.628 & & & \\
\hline & & Stomach & 1.184 & $0.665-2.107$ & 0.567 & & & \\
\hline & & Kidney & 1.581 & $0.636-3.928$ & 0.324 & & & \\
\hline & & Adrenal gland & 1.120 & $0.608-2.060$ & 0.717 & & & \\
\hline & & Coeliac trunk & 3.091 & $1.226-7.797$ & 0.017 & 3.364 & $1.147-9.861$ & 0.027 \\
\hline & & Portal vein & 1.711 & $0.962-3.044$ & 0.067 & 2.275 & $1.221-4.236$ & 0.010 \\
\hline & Intraoperative PRBC & & 2.235 & $1.429-3.498$ & $<0.001$ & 1.998 & $1.229-3.247$ & 0.005 \\
\hline & Intraoperative PRBC (n) & & 1.160 & $1.080-1.245$ & $<0.001$ & & & \\
\hline & Intraoperative FFP & & 1.808 & $1.024-3.191$ & 0.041 & & & \\
\hline & $\begin{array}{l}\text { Operation time } \\
\text { (in minutes) }\end{array}$ & & 1.004 & $1.000-1.009$ & 0.033 & & & \\
\hline \multirow{10}{*}{$\begin{array}{l}\text { Histopathological } \\
\text { results }\end{array}$} & Tumor localization & Including body & 1.053 & $0.691-1.604$ & 0.811 & & & \\
\hline & $\begin{array}{l}\text { Invasion of peripancreatic } \\
\text { tissue }\end{array}$ & & 2.148 & $1.182-3.904$ & 0.012 & & & \\
\hline & \multirow[t]{2}{*}{ T staging } & Tumor size (in cm) & 1.060 & $0.983-1.143$ & 0.132 & & & \\
\hline & & T stage $\geq 3$ & 1.558 & $1.019-2.381$ & 0.040 & & & \\
\hline & \multirow[t]{5}{*}{ Lymph node status } & Lymph nodes ( $n$, total) & 0.985 & $0.958-1.012$ & 0.271 & & & \\
\hline & & Lymph nodes ( $n$, positive) & 1.201 & $1.065-1.355$ & 0.003 & & & \\
\hline & & Lymph node ratio (in \%) & 1.020 & $1.008-1.033$ & 0.002 & 1.022 & $1.009-1.034$ & 0.001 \\
\hline & & $\geq \mathrm{N} 1$ stage & 1.688 & $1.100-2.592$ & 0.017 & & & \\
\hline & & N 2 stage & 2.095 & $1.027-4.274$ & 0.042 & & & \\
\hline & M 1 stage & & 1.381 & $0.825-2.311$ & 0.219 & & & \\
\hline
\end{tabular}


Table 3 Cox regression analysis for identification of risk factors for survival after distal pancreatectomy (Continued)

\begin{tabular}{|c|c|c|c|c|c|c|c|c|}
\hline \multirow[t]{2}{*}{ Variables } & & & \multicolumn{3}{|c|}{ Univariable analysis } & \multicolumn{3}{|c|}{ Multivariable analysis } \\
\hline & & & $\overline{\mathrm{HR}}$ & Cl 95\% & $\overline{p \text { value }}$ & $\overline{H R}$ & Cl 95\% & $\overline{p \text { value }}$ \\
\hline & Grading & Grading $>2$ & 1.266 & $0.826-1.939$ & 0.279 & & & \\
\hline & Resection margin & $\geq R 1$ & 1.892 & $1.219-2.935$ & 0.004 & & & \\
\hline & & R 2 status & 1.008 & $0.464-2.189$ & 0.984 & & & \\
\hline & $\begin{array}{l}\text { AJCC/UICC classification } \\
\text { (8th ed.) }\end{array}$ & $\geq \| I$ & 1.815 & $1.149-2.868$ & 0.011 & & & \\
\hline
\end{tabular}

Bold values indicate statistical significance $(p<0.050)$

ASA American Society of Anesthesiologists, PRBC packed red blood cells, FFP fresh frozen plasma, AJCC American Joint Committee on Cancer, UICC Union for International Cancer Control

meta-analyses on the matter, a large multi-center study by Paye et al. and previously published single-center reports support our observations [10,11,27-30]. Of note, most of these publications included resections for different types of pancreatic tumors, whereas the current study focused explicitly on histologically confirmed pancreatic ductal adenocarcinoma.

The rate of arterial and venous resections in our patient cohort was comparable to previous publications; however, reports on the effects of vascular resections in case of distal pancreatectomy are conflicting [10, 27]. In general, the need for arterial resections to achieve tumor-free resection margins in case of pancreatic adenocarcinoma is seen critical or even as a contraindication for surgery, as it is associated with increased morbidity and mortality [13]. In the current study, resection of the coeliac trunk was associated with a considerable increase in postoperative mortality with two of five patients dying in the postoperative course and a resulting median survival of only 3.52 months. Nonetheless, a recent systematic review and meta-analysis by Gong et al. revealed that distal pancreatectomy with en bloc celiac axis resection can result in favorable survival, despite increased postoperative morbidity, and improved quality of life in selected patients, especially when compared to palliative treatment [31].

As a result of refined surgical techniques, simultaneous resections of the portal and/or the superior mesenteric vein are generally regarded as safe and can facilitate longterm survival $[13,32]$. Data on the effect of such venous resections in case of distal pancreatectomy is scarce, and recommendations result mostly from reports on pancreatic head carcinoma [33-36]. Interestingly, recent metaanalyses as well as large single center studies have shown rather discouraging results [35-37]. In the current study, simultaneous resection of the portal vein resulted in a median survival of only 7.56 months and only one patient survived more than 5 years. Previous publications did not identify resections of the mesenteric-portal axis as risk factors for survival in case of distal pancreatectomy; however, missing details of the extent of venous resection or mainly minor resections (such as wedge or tangential resections) limit the significance and comparability of such observations $[11,27]$. In our study, all but one patient underwent segmental resections of the portal vein due to macroscopically suspected invasion. Such resections, unlike tangential resections, were recently identified as an independent risk factor for postoperative survival after resection of pancreatic adenocarcinoma by Serenari et al. [38].

Further prognostic factors identified in the current study were intraoperative blood transfusions, which were demonstrated to result in adverse oncologic effects such as shorter disease-free survival in the past, and a higher lymph node ratio [39]. The latter has been confirmed by several authors including a systematic review for pancreatic adenocarcinoma in general. Thus, apart from the classical nodal status, the lymph node ratio should be an inherent part when planning and optimizing postoperative adjuvant strategies [23, 40-45].

The incidence of clinically relevant pancreatic fistulas was comparable to reports of the past [46]. Although we observed a trend towards more fistulas in patients after multivisceral resection (30.4\%) and especially after simultaneous partial colectomy (50.0\%), multivariable analysis did not identify extended surgery as an independent risk factor. This is consistent with reports from previous single-center studies [30, 47]. Recently, Ecker et al. analyzed 2026 consecutive distal pancreatectomies and identified risk factors such as young age, obesity, non-malignant histology, or concomitant splenectomy, among others; however, the authors concluded that despite the considerable size of the study, postoperative pancreatic fistulas cannot be reliably predicted, since individual surgeon performance, applied techniques, and patient-specific pancreatic texture limit the significance of clinical applicability of mentioned observations [46].

Despite long follow-up durations-only one surviving patient was followed up less than 5 years-the retrospective nature of the study and the limited number of patients hamper definitive conclusions. Further limitations of our study are missing information on disease recurrence and systemic therapy, especially as the latter is of increasing 
importance in a more and more multimodal approach for pancreatic cancer $[48,49]$. Although reliable data on this matter is still scarce, a review of resections in patients with oligometastatic pancreatic disease underlines the importance of standardizing neoadjuvant and adjuvant chemo(radio)therapy strategies and defines a response towards neoadjuvant systemic treatment as important patient selection criterion along with an adequate performance status as well as resectability of the primary tumor and the metastases [50]. Larger patient cohorts, meta-analyses, and prospective trials are urgently required to confirm our observations in this lethal disease.

\section{Conclusions}

Our data indicates that multivisceral resection in case of advanced left-sided pancreatic adenocarcinoma is justified, as it is not associated with increased mortality and can even facilitate long-term survival, albeit with an increase in postoperative morbidity. However, vascular resections are associated with a dismal prognosis and should be performed after thorough consideration and only in selected patients.

\section{Supplementary information}

Supplementary information accompanies this paper at https://doi.org/10 1186/s12957-020-01973-X.

Additional file 1: Supplementary Table 1. Overview of the different types of resections in the investigated cohort. Supplementary Table 2. Logistic regression analysis identifying risk factors for clinically relevant pancreatic fistulas after distal pancreatectomy.

Additional file 2: Supplementary Table 3.1. Overview of patients undergoing standard distal pancreatectomy including selected variables sorted by year of surgery. Supplementary Table 3.2. Overview of patients undergoing multivisceral resection including selected variables sorted by year of surgery.

\section{Abbreviations}

AJCC: American Joint Committee on Cancer; ASA: American Society of Anesthesiologists; UICC: Union for International Cancer Control; CA199: Carbohydrate antigen 19-9; CEA: Carcinoembryonic antigen; PRBC: Packed red blood cells; OR: Odds ratio; HR: Hazard ratio; Cl: Confidence interval; PV: Portal vein; LNR: Lymph node ratio

\section{Acknowledgements}

Not applicable.

\section{Authors' contributions}

$O B, M W$, and $G G$ designed the study. OB, AS, and GG extracted and collected data. $O B, A K$, and $G G$ analyzed and interpreted the data. $O B$ and GG drafted the manuscript. AS, JK, and MW critically revised the manuscript. All authors read and approved the final manuscript.

\section{Funding}

This study did not receive any type of funding. Open access funding provided by Projekt DEAL.

\section{Availability of data and materials}

The datasets used and/or analyzed during the current study are available from the corresponding author on reasonable request.

\section{Ethics approval and consent to participate}

Patients provided informed consent that their data may be used for scientific purposes at the time of hospital admission, which is the general policy of our institution. The ethical committee of Hannover Medical School stated that no further approval for retrospective analyses is needed. Patient records and patient data were anonymized and de-identified prior to analysis.

\section{Consent for publication}

Not applicable.

\section{Competing interests}

The authors declare that they have no competing interests.

Received: 1 April 2020 Accepted: 28 July 2020

Published online: 20 August 2020

\section{References}

1. McGuigan A, Kelly P, Turkington RC, Jones C, Coleman HG, McCain RS Pancreatic cancer: a review of clinical diagnosis, epidemiology, treatment and outcomes. World J GastroenterolBaishideng Publishing Group Co., Limited. 2018:4846-61.

2. Rawla P, Sunkara T, Gaduputi V. Epidemiology of pancreatic cancer: global trends, etiology and risk factors. World J OncolElmer Press, Inc. 2019;10:10-27.

3. Abbassi $\mathrm{R}$, Algül $\mathrm{H}$. Palliative chemotherapy in pancreatic cancer-treatment sequences. Transl. Gastroenterol. Hepatol. AME Publishing Company; 2019.

4. Paniccia A, Hosokawa P, Henderson W, Schulick RD, Edil BH, McCarter MD, et al. Characteristics of 10-year survivors of pancreatic ductal adenocarcinoma. JAMA SurgAmerican Medical Association. 2015;150:701-10.

5. Dusch N, Weiss C, Ströbel P, Kienle P, Post S, Niedergethmann M. Factors predicting long-term survival following pancreatic resection for ductal adenocarcinoma of the pancreas: 40 years of experience. J Gastrointest SurgSpringer New York LLC. 2014;18:674-81.

6. Artinyan A, Soriano PA, Prendergast C, Low T, Ellenhorn JDI, Kim J. The anatomic location of pancreatic cancer is a prognostic factor for survival. HPB. Taylor and Francis Ltd.; 2008. p. 371-6.

7. Ducreux M, Cuhna AS, Caramella C, Hollebecque A, Burtin P, Goéré D, et al. Cancer of the pancreas: ESMO clinical practice guidelines for diagnosis, treatment and follow-up. Ann OncolOxford University Press. 2015;26:v56-68.

8. van Erning FN, Mackay TM, van der Geest LGM, Groot Koerkamp B, van Laarhoven HWM, Bonsing BA, et al. Association of the location of pancreatic ductal adenocarcinoma (head, body, tail) with tumor stage, treatment, and survival: a population-based analysis. Acta Oncol (Madr)Taylor and Francis Ltd. 2018;57:1655-62.

9. Birnbaum DJ, Bertucci F, Finetti P, Birnbaum D, Mamessier E. Head and body/tail pancreatic carcinomas are not the same tumors. Cancers (Basel)MDPI AG. 2019:11.

10. Malinka T, Klein F, Andreou A, Pratschke J, Bahra M. Distal pancreatectomy combined with multivisceral resection is associated with postoperative complication rates and survival comparable to those after standard procedures. J Gastrointest SurgSpringer New York LLC. 2018;22:1549-56.

11. Panzeri F, Marchegiani G, Malleo G, Malpaga A, Maggino L, Marchese T, et al. Distal pancreatectomy associated with multivisceral resection: results from a single Centre experience. Langenbeck's Arch SurgSpringer Verlag. 2017;402:457-64.

12. Delpero JR, Sauvanet A. Vascular resection for pancreatic cancer: 2019 French recommendations based on a literature review from 2008 to 6-2019. Front OncolFrontiers Media S.A. 2020:40.

13. Bockhorn M, Uzunoglu FG, Adham M, Imrie C, Milicevic M, Sandberg AA, et al. Borderline resectable pancreatic cancer: a consensus statement by the international study Group of Pancreatic Surgery (ISGPS). Surg (United States)Mosby Inc. 2014;155:977-88.

14. Tol JAMG, Gouma DJ, Bassi C, Dervenis C, Montorsi M, Adham M, et al. Definition of a standard lymphadenectomy in surgery for pancreatic ductal adenocarcinoma: a consensus statement by the international study group on pancreatic surgery (ISGPS). Surg (United States)Mosby Inc. 2014;156:591-600.

15. Hartwig W, Vollmer CM, Fingerhut A, Yeo CJ, Neoptolemos JP, Adham M, et al. Extended pancreatectomy in pancreatic ductal adenocarcinoma: definition and consensus of the International Study Group for Pancreatic Surgery (ISGPS). Surg (United States). Mosby Inc.; 2014;156:1-14. 
16. Mayhew D, Mendonca $V$, Murthy BVS. A review of ASA physical status historical perspectives and modern developments. AnaesthesiaBlackwell Publishing Ltd. 2019;74:373-9.

17. World Health Organization (WHO). Haemoglobin concentrations for the diagnosis of anaemia and assessment of severity. Vitamin and Mineral Nutrition Information System. Geneva, World Health Organization, 2011 (WHO/NMH/NHD/MNM/11.1) https://www.who.int/vmnis/indicators/ haemoglobin.pdf. Accessed 20 July 2020.

18. Bassi C, Marchegiani G, Dervenis C, Sarr M, Abu Hilal M, Adham M, et al. The 2016 update of the International Study Group (ISGPS) definition and grading of postoperative pancreatic fistula: 11 years after. Surg. (United States). Mosby Inc.; 2017. p. 584-91.

19. Cong L, Liu Q, Zhang R, Cui M, Zhang X, Gao X, et al. Tumor size classification of the 8th edition of TNM staging system is superior to that of the 7th edition in predicting the survival outcome of pancreatic cancer patients after radical resection and adjuvant chemotherapy. Sci Rep. Nature Publishing Group; 2018;8:1-9.

20. Von Hoff DD, Ervin T, Arena FP, Chiorean EG, Infante J, Moore M, et al. Increased survival in pancreatic cancer with nab-paclitaxel plus gemcitabine. N Engl J MedMassachussetts Medical Society. 2013;369:1691-703.

21. Ruess DA, Makowiec F, Chikhladze S, Sick O, Riediger H, Hopt UT, et al. The prognostic influence of intrapancreatic tumor location on survival after resection of pancreatic ductal adenocarcinoma visceral and general surgery. BMC Surg. BioMed Central Ltd.; 2015;15:123.

22. Seppänen $H$, Juuti $A$, Mustonen $H$, Haapamäki C, Nordling S, CarpelanHolmström M, et al. The results of pancreatic resections and long-term survival for pancreatic ductal adenocarcinoma: a single-institution experience. Scand J Surg. SAGE Publications Inc.; 2017;106:54-61.

23. Riediger H, Keck T, Wellner U, Zur Hausen A, Adam U, Hopt UT, et al. The lymph node ratio is the strongest prognostic factor after resection of pancreatic cancer. J Gastrointest Surg. 2009;13:1337-44.

24. Sheng W, Dong M, Wang G, Shi X, Gao W, Wang K, et al. The diversity between curatively resected pancreatic head and body-tail cancers based on the 8th edition of AJCC staging system: a multicenter cohort study. BMC Cancer. BioMed Central Ltd.; 2019;19.

25. Demir IE, Jäger C, Schlitter MM, Konukiewitz B, Stecher L, Schorn S, et al. R0 versus $\mathrm{R} 1$ resection matters after pancreaticoduodenectomy, and less after distal or total pancreatectomy for pancreatic cancer. Ann SurgLippincott Williams and Wilkins. 2017;268:1058-68.

26. Petrucciani N, Debs T, Nigri G, Giannini G, Sborlini E, Kassir R, et al. Pancreatectomy combined with multivisceral resection for pancreatic malignancies: is it justified? Results of a systematic review; 2018.

27. Paye F, Micelli Lupinacci R, Bachellier P, Boher JM, Delpero JR. Distal pancreatectomy for pancreatic carcinoma in the era of multimodal treatment. Br J SurgJohn Wiley and Sons Ltd. 2015;102:229-36.

28. Irani JL, Ashley SW, Brooks DC, Osteen RT, Raut CP, Russell S, et al. Distal pancreatectomy is not associated with increased perioperative morbidity when performed as part of a multivisceral resection. J Gastrointest SurgSpringer. 2008;12:2177-82.

29. Kleeff J, Diener MK, Z'graggen K, Hinz U, Wagner M, Bachmann J, et al. Distal pancreatectomy. Ann Surg. 2007;245:573-82.

30. Reeh M, Nentwich MF, Bogoevski D, Koenig AM, Gebauer F, Tachezy M, et al. High surgical morbidity following distal pancreatectomy: still an unsolved problem. World J SurgSpringer. 2011;35:1110-7.

31. Gong H, Ma R, Gong J, Cai C, Song Z, Xu B. Distal pancreatectomy with en bloc celiac axis resection for locally advanced pancreatic cancer: a systematic review and meta-analysis. Med (United States). Lippincott Williams and Wilkins; 2016;95.

32. Hirono S, Kawai M, Tani M, Okada Kl, Miyazawa M, Shimizu A, et al. Indication for the use of an interposed graft during portal vein and/or superior mesenteric vein reconstruction in pancreatic resection based on perioperative outcomes. Langenbeck's Arch SurgSpringer Verlag. 2014;399:461-71.

33. Yu XZ, Li J, Fu DL, Di Y, Yang F, Hao SJ, et al. Benefit from synchronous portal-superior mesenteric vein resection during pancreaticoduodenectomy for cancer: a meta-analysis. Eur. J. Surg. Oncol. W.B. Saunders Ltd; 2014. p. 371-8.

34. Müller SA, Hartel M, Mehrabi A, Welsch T, Martin DJ, Hinz U, et al. Vascular resection in pancreatic cancer surgery: survival determinants. J Gastrointest SurgSpringer. 2009;13:784-92.
35. Giovinazzo F, Turri G, Katz MH, Heaton N, Ahmed I. Meta-analysis of benefits of portal-superior mesenteric vein resection in pancreatic resection for ductal adenocarcinoma. Br J SurgJohn Wiley and Sons Ltd. 2016:179-91.

36. Bell R, Te Ao B, Ironside N, Bartlett A, Windsor JA. Pandanaboyana S. Metaanalysis and cost effective analysis of portal-superior mesenteric vein resection during pancreatoduodenectomy: impact on margin status and survival. Surg OncolElsevier Ltd. 2017:53-62.

37. Mierke F, Hempel S, Distler M, Aust DE, Saeger HD, Weitz J, et al. Impact of portal vein involvement from pancreatic cancer on metastatic pattern after surgical resection. Ann Surg OncolSpringer New York LLC. 2016;23:730-6.

38. Serenari M, Ercolani G, Cucchetti A, Zanello M, Prosperi E, Fallani G, et al. The impact of extent of pancreatic and venous resection on survival for patients with pancreatic cancer. Hepatobiliary Pancreat Dis Int. Elsevier (Singapore) Pte Ltd; 2019;18:389-94.

39. Hwang HK, Jung MJ, Lee SH, Kang CM, Lee WJ. Adverse oncologic effects of intraoperative transfusion during pancreatectomy for left-sided pancreatic cancer: the need for strict transfusion policy. J Hepatobiliary Pancreat SciBlackwell Publishing Asia. 2016;23:497-507.

40. Mirkin KA, Hollenbeak CS, Wong J. Greater lymph node retrieval and lymph node ratio impacts survival in resected pancreatic cancer. J Surg ResAcademic Press Inc. 2017;220:12-24.

41. You MS, Lee SH, Choi YH, Shin BS, Paik WH, Ryu JK, et al. Lymph node ratio as valuable predictor in pancreatic cancer treated with $\mathrm{RO}$ resection and adjuvant treatment. BMC Cancer. BioMed Central Ltd:; 2019;19:952.

42. Karaca CA, Coker A. Prognostic value of metastatic lymph node ratio in pancreatic cancer. Indian J Surg OncolSpringer. 2019;10:50-4.

43. Zhan HX, Xu JW, Wang L, Zhang GY, Hu SY. Lymph node ratio is an independent prognostic factor for patients after resection of pancreatic cancer. World J Surg Oncol. BioMed Central Ltd.; 2015;13:105.

44. Yamada S, Fujii T, Hirakawa A, Kanda M, Sugimoto H, Kodera Y. Lymph node ratio as parameter of regional lymph node involvement in pancreatic cancer. Langenbeck's Arch Surg. Springer Verlag; 2016;401:1143-52.

45. Elshaer M, Gravante G, Kosmin M, Riaz A, Al-Bahrani A. A systematic review of the prognostic value of lymph node ratio, number of positive nodes and total nodes examined in pancreatic ductal adenocarcinoma. Ann. R. Coll. Surg. Engl. Royal College of Surgeons of England; 2017. p. 101-6.

46. Ecker BL, McMillan MT, Allegrini V, Bassi C, Beane JD, Beckman RM, et al. Risk factors and mitigation strategies for pancreatic fistula after distal pancreatectomy: analysis of 2026 resections from the international, multiinstitutional distal pancreatectomy study group. Ann SurgLippincott Williams and Wilkins. 2019:269:143-9.

47. Seeliger H, Christians S, Angele MK, Kleespies A, Eichhorn ME, Ischenko I, et al. Risk factors for surgical complications in distal pancreatectomy. Am J Surg. 2010;200:311-7.

48. Hajatdoost L, Sedaghat K, Walker EJ, Thomas J, Kosari S. Chemotherapy in pancreatic cancer: a systematic review. Med. MDPI AG; 2018.

49. Rangarajan K, Pucher PH, Armstrong T, Bateman A, Hamady ZZR. Systemic neoadjuvant chemotherapy in modern pancreatic cancer treatment: a systematic review and meta-analysis. Ann. R. Coll. Surg. Engl. Royal College of Surgeons of England; 2019. p. 453-62.

50. Lu F, Poruk KE, Weiss MJ. Surgery for oligometastasis of pancreatic cancer. Chinese J. Cancer Res. AME Publishing Company. 2015:358-67.

\section{Publisher's Note}

Springer Nature remains neutral with regard to jurisdictional claims in published maps and institutional affiliations.

Ready to submit your research? Choose BMC and benefit from:

- fast, convenient online submission

- thorough peer review by experienced researchers in your field

- rapid publication on acceptance

- support for research data, including large and complex data types

- gold Open Access which fosters wider collaboration and increased citations

- maximum visibility for your research: over $100 \mathrm{M}$ website views per year

At BMC, research is always in progress.

Learn more biomedcentral.com/submissions 